\title{
La industria del gas natural y su regulación en América Latina
}

\section{Humberto Campodónico}

Consultor de la CEPAL. Investigador Principal, Centro de Estudios

y Promoción del

Desarrollo (DESCO)

Lima, Perú, y

Director de la Unidad

de Postgrado,

Facultad de Economía,

Universidad Mayor de

San Marcos de Lima
Este artículo analiza los regímenes de regulación de los mercados de gas natural en Argentina, Bolivia, Colombia, Chile y México, los países latinoamericanos que más han avanzado en este campo, y también presenta algunas informaciones sobre países que, al momento de redactarlo, no habían definido aún su régimen regulatorio, como Perú y Venezuela. Para comenzar se describe el panorama mundial de la industria de gas natural y se define el lugar que le corresponde en él a América Latina. Luego se estudian las modificaciones que experimentaron los regímenes regionales de la industria del gas natural en el decenio de 1990, en particular en el tratamiento de la exploración y la producción, y en el procesamiento industrial, el transporte y la comercialización. Se detallan en seguida las características principales de los mercados de gas natural en los países estudiados y los sistemas que se han establecido para reestructurar la industria, dándole especial importancia al papel del gas natural en la integración energética regional. Por último, se analizan las características principales de la regulación del gas natural y los principios que la sustentan; la estructura y atribuciones de los entes reguladores; los mecanismos de fijación de precios de explotación, transporte y distribución; las modalidades de subsidio, y el régimen tributario. 
I

\section{El comportamiento de la demanda}

y las proyecciones del consumo

mundial de gas natural

En los últimos veinte años la demanda mundial de gas natural ha crecido con rapidez mayor que la de los otros combustibles fósiles (petróleo y carbón). En el período 1971-1991, su tasa anual de crecimiento llegó a $3.3 \%$ frente al $2.1 \%$ para el carbón y el $1.4 \%$ para el petróleo. ${ }^{1}$

El mayor dinamismo del consumo del gas natural se explica principalmente por lo siguiente:

- Gracias a la abundancia de reservas de gas natural, la capacidad de oferta podría mantenerse por muchos años; además, esas reservas no se encuentran muy concentradas geográficamente (en cambio, cerca de dos tercios de las reservas mundiales disponibles de petróleo se encuentran en el Medio Oriente).

- Los avances tecnológicos han abaratado los costos de transporte desde las fuentes de producción hasta los centros de consumo. ${ }^{2}$

- En los últimos decenios ha cobrado mayor importancia la conservación del medio ambiente, lo que favorece el consumo del gas natural, que emite menos dióxido de carbono que el petróleo.

- El crecimiento de los mercados energéticos en los países emergentes ha hecho más atractiva la in-

El presente artículo se basa en la publicación sobre el tema aparecido en la serie Medio Ambiente y Desarrollo, $\mathrm{N}^{\circ}$ 9, de la División de Medio Ambiente y Desarrollo de la CEPAL.

${ }^{1}$ Véase un análisis detallado en Estados Unidos, Departamento de Energía (1995), y ocDE, Organismo Internacional de Energía (1995).

${ }^{2}$ El transporte de gas natural es más complicado que el del petróleo y el carbón, que se extraen en estado líquido o sólido respectivamente, puesto que debe mantenerse en una unidad completamente cerrada para su manipulación. Los gasoductos son los medios más comunes de transporte, pero cuando la distancia geográfica es muy grande, su utilización es no sólo difícil sino muy costosa. El gas debe ser transportado por tanto en barcos refrigerados y convertido a temperaturas bajas a estado líquido — gas natural líquido- para ser colocado en los mercados de destino, siendo objeto posteriormente de una "regasificación" para ser distribuido por gasoducto. El problema con el gas natural líquido es que el proceso de conversión, el costo de los propios barcos, la manipulación y las instalaciones especiales le añaden un costo considerable, reduciendo su atractivo económico (Estados Unidos, Departamento de Energía, 1995). versión para los operadores internacionales y nacionales.

- La política de seguridad energética de los países de la OCDE apunta a reducir la dependencia del petróleo y del gas natural importado, sobre todo del Medio Oriente.

La mayor proporción del consumo de gas natural se concentra en los países de la OCDE, y en los países de la ex Unión Soviética y las economías en transición de Europa oriental, que representan respectivamente el $48 \%$ y el $36 \%$ de la demanda mundial. Entre las regiones en desarrollo, el Medio Oriente y América Latina cubren, cada uno, cerca de $5 \%$ de la demanda mundial. Les siguen Asia oriental y Asia meridional con $2.1 \%$ y $1.4 \%$, respectivamente, mientras que Africa satisface el $1.45 \%$ y la República Popular China el $0.75 \%$ del consumo mundial.

El petróleo, el carbón y el gas natural — todos combustibles fósiles- abastecían el $91 \%$ del consumo mundial de energía a mediados del decenio de 1990: $38.8 \%$ el petróleo, $28.4 \%$ el carbón y $23.6 \%$ el gas natural. Las proyecciones del consumo de energía para el período 1995-2020 indican que ese patrón continuaría (OCDE, Organismo Internacional de Energía, 1995). En efecto, la demanda de gas natural seguirá creciendo a un ritmo superior al que registrarán el carbón y el petróleo, con tasas de $2.4 \%, 2.1 \%$ y $0.8 \%$ respectivamente. Siendo así, la proporción del consumo total de energía abastecido por estas tres fuentes subiría hasta cubrir $92.2 \%$ de la demanda mundial de energía, sobre todo gracias al aumento del consumo de gas natural, cuya contribución llegaría al 25.2\%; la participación del carbón y del petróleo se mantendría estable (gráfico 1).

Según la ocDE (1995), los países en desarrollo registrarían la mayor tasa de crecimiento del consumo con un $5.6 \%$, seguidos por los países de la OCDE con $2.1 \%$ y por los de la ex Unión Soviética y las economías en transición de Europa del este, con un $0.8 \%$. China y el Medio Oriente alcanzarían la tasa de crecimiento más alta (8 y $7 \%$, respectivamente). Para América Latina se proyecta una tasa de $3 \%$, aunque 
GRAFICO 1

\section{Mundo: Consumo de energía en 1995 y proyecciones al 2020 (Porcentajes)}

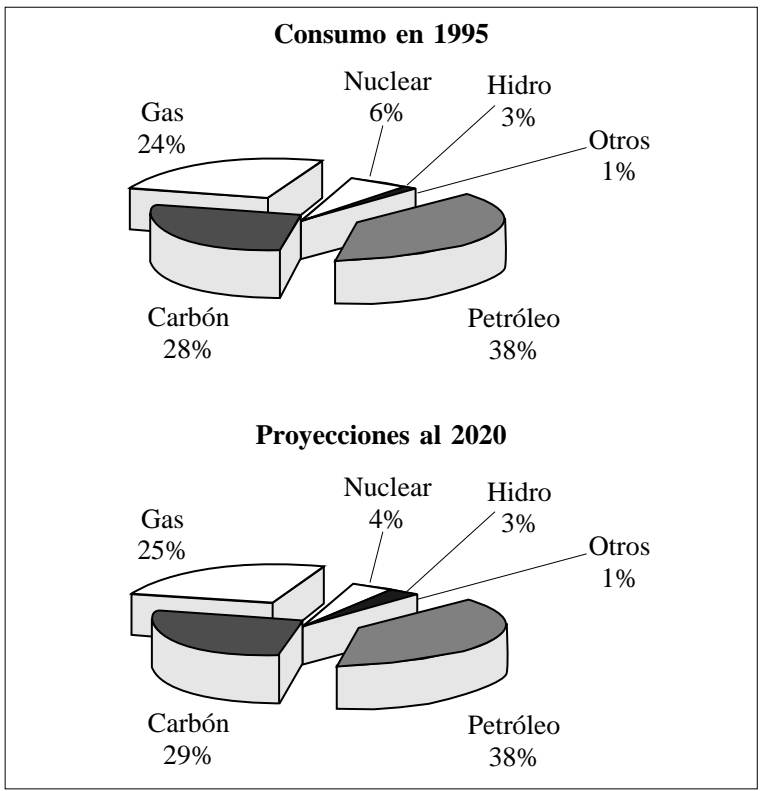

Fuente: OCDE, Organismo Internacional de Energía (1995).

otras fuentes la estiman en 5\%. ${ }^{3}$ (OCDE, Organismo Internacional de Energía, 1995).

Estas tasas tan elevadas se traducirán en el próximo siglo en modificaciones importantes de la estructura de la demanda mundial de gas natural. Los países de la OCDE continuarían siendo el principal mercado de consumo, pero su participación en el total mundial bajará de 48 a 45\%. La ex URSs y las economías en transición de Europa del este registrarán una fuerte caída de casi $10 \%$, para situarse en torno al $27 \%$, mientras que la participación en el consumo mundial de los países en desarrollo subirá en $12 \%$ (al $27.8 \%$ del total).

Las reservas de los países de la región se han cuadruplicado en los últimos 25 años (gráfico 2). Venezuela, México y Argentina representan el 89\% del total; Venezuela posee más de la mitad de las reservas de la región (3962 x $10^{9}$ metros cúbicos). En Brasil, Colombia, Ecuador y Perú han aumentado las reservas, pero en volúmenes bastante menores.

${ }^{3}$ Existen discrepancias con respecto a la tasa de crecimiento de la demanda de gas natural en América Latina. DRI McGraw-Hill pronostica que para el período 1995-2015, la tasa de crecimiento de la región sería de $5.1 \%$ anual, lo que coincide con las proyecciones de Amoco International (Oil \& Gas Journal, varios números).
Pocos países de la región son productores tradicionales de gas natural, producción que en la mayoría de los casos está vinculada con la de petróleo. La producción de gas natural por países muestra un patrón similar al que registran las reservas petrolíferas. Venezuela, México y Argentina aportan el $84 \%$ de la producción regional de gas natural (gráfico 3).

La demanda final de energía de los países de la región se cubre en $42 \%$ con petróleo y en $21 \%$ con electricidad (centrales hidráulicas, nucleares y geotérmicas). La participación del gas natural es de $18 \%$, mayor que la de la biomasa (15\%) y el carbón (4\%). Sin embargo, ese promedio encubre fuertes disparidades dentro de la región ya que Argentina, Brasil y Venezuela representan el $92 \%$ de la demanda regional (gráfico 4).

Sigue siendo notable la diferencia entre la demanda final de gas natural y el consumo en usos intermedios. En 1995, la estructura de la oferta total de gas natural (consumo final más consumo intermedio) por sectores en América Latina (cuadro 1) muestra que sólo el $33 \%$ del gas natural se destinaba al consumo final

GRAFICO 2

\section{Reservas probadas de gas natural en América Latina (109 metros cúbicos)}

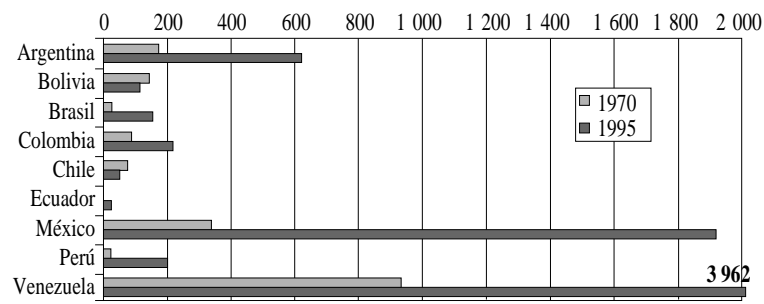

Fuente: OLADE, 1997a.

GRAFICO 3

Producción de gas natural en América Latina (10 metros cúbicos)

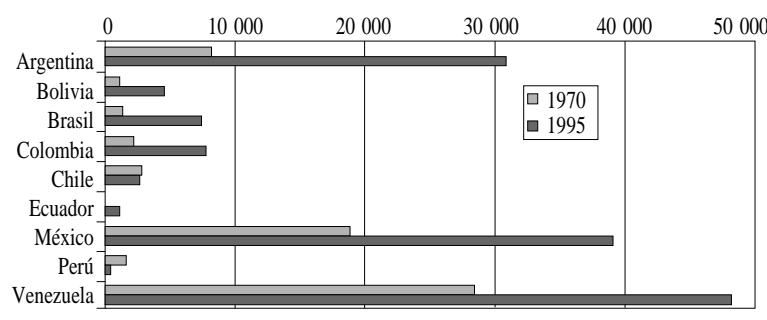

Fuente: OLADE, 1997a. 
GRAFICO 4

Consumo de gas natural en América Latina (10 metros cúbicos)

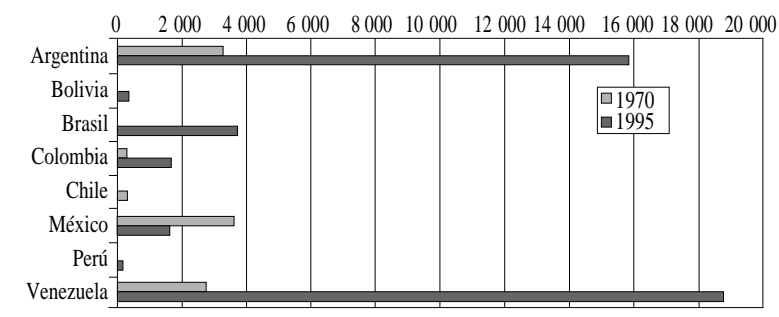

Fuente: OLADE, 1997a.

energético (42 330 millones de metros cúbicos), principalmente en el sector industrial (22\%), residencial y otros $(5.9 \%)$ y en menor medida en el sector comercial, no energético y de transporte.

Las dos terceras partes de la oferta total de 141025 millones de metros cúbicos $\left(\mathrm{MMm}^{3}\right)$ se destinan a usos intermedios, como plantas de tratamiento de gas para obtener gas licuado de petróleo (GLP) y gasolinas $(32.8 \%)$, consumo propio $(17.1 \%)$, centrales térmicas y autoproductores $(15.8 \%)$, correspondiendo el resto a refinerías y otros usos y pérdidas.
CUADRO 1

\section{América Latina, 1996: Abastecimiento de gas según uso \\ (Porcentajes)}

Plantas de tratamiento de gas 32.8

Industria 22.0

Consumo propio 17.1

Plantas de energía

Residencial y otro

No energía

Productores particulares

Comercial

Transporte

Otros centros

Refinerías

13.2

Total

100.0

Total en millones de $\mathrm{m}^{3}$

141025

Fuente: Kurtz (1997).

Todo indica, sin embargo, que la demanda final de gas natural tenderá a crecer en los próximos años, habida cuenta de los importantes proyectos energéticos en cartera y la orientación de algunas políticas gubernamentales que están promoviendo la masificación de su consumo.

\section{II}

\section{Reformas a la industria del gas natural}

En lo que va del decenio de 1990 se han introducido en la mayoría de los países analizados importantes reformas legislativas en los regímenes jurídicos de la exploración y la explotación de hidrocarburos (Argentina, Bolivia, Colombia y Chile). Las reformas afectan tanto al petróleo como al gas natural, ya que presentan características similares de exploración y explotación, pero se precisan en esas reformas las particularidades que presenta el gas natural. ${ }^{4}$

En México no se reformó la legislación petrolera en sí, pero se introdujeron importantes modificaciones en las normas sobre transporte, almacenamiento y distribución del gas natural y se conservó el monopolio de la empresa estatal Petróleos Mexicanos (PEMEX) en la exploración y explotación del gas natural.

\footnotetext{
${ }^{4}$ Para un análisis más detallado del sector hidrocarburos en diez países de la región, véase Campodónico, 1996.
}

El origen y las motivaciones de las reformas que se han llevado a cabo en la mayoría de los países de la región se enmarcan en el proceso de globalización de la economía mundial que favorece la liberalización de los mercados, la eliminación de los monopolios estatales, la promoción de la inversión privada y la reducción de la actividad empresarial del Estado. Sin embargo, se observan diferencias importantes en la aplicación de estas reformas, por la influencia de factores tales como la dotación de reservas y la relación producción/consumo de los derivados del petróleo y el gas natural, el tamaño del mercado interno, el nivel tecnológico y la experiencia en la industria petrolera y del gas natural.

\section{Reformas al régimen de exploración y explotación de petróleo y gas natural}

Los regímenes de exploración y explotación se aplican tanto al petróleo como al gas natural. Por lo general, las diferencias se presentan en los montos de las 
regalías y en los mecanismos para la determinación del precio de venta del gas natural de primera mano, tomando en cuenta que el gas natural no tiene las mismas características de bien transable a nivel internacional que el petróleo.

Casi todos los países considerados en este artículo - a excepción de México que mantiene el monopolio estatal de PEMEX - han reformado sus regímenes de contratación para la exploración y la explotación de petróleo crudo y gas natural, con el objeto de atraer capital de riesgo para ampliar la disponibilidad de reservas e incrementar las exportaciones o para reducir las importaciones, a fin de obtener un saldo neto de divisas más favorable en sus transacciones energéticas.

La mayoría de los países analizados consideran que son necesarios mayores incentivos para la inversión ante la fuerte competencia que existe por atraer capitales de riesgo en las actuales condiciones de sobreoferta de crudo, bajos precios internacionales del petróleo, innovaciones tecnológicas que permiten un mayor aprovechamiento de las reservas existentes y la eliminación de las barreras a la inversión extranjera en zonas con filiación petrolífera importante (Rusia, China).

En lo esencial, las reformas han incidido en la disminución de las tasas impositivas a los operadores privados y en la libre disponibilidad comercial del crudo y del gas natural extraído; además se tiende a suprimir el monopolio público en las etapas extractivas. En Argentina, Bolivia, Colombia, Chile y Perú, en que no había restricciones a la inversión extranjera, los incentivos se han concentrado en los aspectos siguientes:

- Condiciones más flexibles en aspectos técnicos de los contratos: ampliación de los plazos de exploración, eliminación de la obligación de perforar pozos exploratorios, mayor plazo de retención de las áreas en exploración, etc.

- Mayor participación en la producción de petróleo y gas natural u otorgamiento de la propiedad del petróleo y del gas natural a los operadores privados.

- Libre importación y exportación del petróleo, eliminándose, en algunos casos, la obligación de abastecimiento del mercado interno.

- Mayor rentabilidad de los contratos por la baja de las tasas del impuesto a la renta y a las remesas (en algunos casos se eliminó el impuesto a las remesas); la internación temporal de maquinaria (lo que implica el no pago de aranceles), la depreciación acelerada, y otras medidas de exoneración o reducción de tributos.

- Mayores garantías a la inversión extranjera, mediante la suscripción de contratos de estabilidad tributaria, garantía de disponibilidad de divisas, posibilidad de recurrir al arbitraje internacional, entre otros.

\section{Reformas en el procesamiento industrial, el transporte y la comercialización}

En todos los países analizados se ha modificado la legislación que rige las actividades de transporte, distribución y comercialización de gas natural. Las reformas tienden a suprimir las barreras a la inversión privada en una actividad que, en muchos países, estaba reservada para las empresas estatales. Se ha favorecido la desregulación de los mercados internos (eliminación de subsidios y control de precios) y se ha permitido el libre comercio - interno y externo- de petróleo y gas natural.

Los precios en boca de pozo del gas natural para el mercado interno han tendido a fijarse en general de acuerdo con los precios internacionales. Sin embargo, la carencia de precios internacionales de referencia para el gas natural - como los que existen para el petróleo- ha aconsejado a algunos entes reguladores a establecer precios de base o precios iniciales para la venta del gas de primera mano.

En todos los países analizados, los gasoductos pueden ser construidos y operados tanto por empresas estatales como por empresas privadas. En Argentina, Bolivia, Colombia, Chile y Perú no existían restricciones a la participación privada en este rubro, lo que fue reafirmado y ampliado con los cambios legislativos de los años noventa. La novedad radica en la supresión de las barreras que existían en México para que los operadores privados participaran en el transporte, distribución y comercialización del gas natural.

En casi todos los países analizados se han dictado disposiciones jurídicas para regular los mercados de gas natural y las actividades de las compañías privadas en el transporte, la distribución y la comercialización del gas, confiriéndole un estatuto de servicio público. 


\section{III}

\section{Sistemas de reestructuración de la industria del gas natural en América Latina}

Los sistemas de reestructuración de las industrias gasíferas están estrechamente vinculados, en los países que se analizan, con el proceso de liberalización de los mercados y la creciente participación del sector privado, sobre todo de los grandes operadores internacionales.

Las características nacionales de reservas, producción y consumo de gas natural, han determinado estructuras diferenciadas en lo que respecta al mercado interno de cada país. Hay también diferencias en cuanto a planes de expansión, ya sea por ampliación del mercado interno o por conexión con otros países de la región. Por lo tanto, no existe un modelo único de reestructuración, pese a que es común la tendencia liberalizadora en favor de la empresa privada.

\section{Argentina: un país con un mercado maduro}

En la Argentina el consumo de gas natural presenta una gran difusión. Argentina cuenta con la mayor red de gasoductos para transporte y distribución $(50000 \mathrm{~km})$ de la región. El gas natural constituye la segunda fuente en importancia para la generación eléctrica en plantas térmicas después del petróleo residual (fuel oil). Se prevé que este combustible penetrará aún más en el mercado y reemplazará volúmenes importantes de combustibles líquidos, con mejores rendimientos en las plantas térmicas.

En 1992 y 1993 se privatizaron las empresas YPF y Gas del Estado. Con la privatización de YPF toda la producción de gas natural quedó en manos de empresas privadas, las cuales disponen libremente de ella. Tres empresas productoras concentran cerca del 81\% de la oferta de gas en Argentina: yPF, Pérez Companc y Tecpetrol (64, 11 y 6\%, respectivamente).

Con la privatización de Gas del Estado en 1992 se produjo la separación de las actividades de transporte y distribución. La privatización supuso la venta de los dos gasoductos más importantes a empresas distintas - Transportadora de Gas del Norte (TGN) y Transportadora de Gas del Sur (TGS) — las mismas que cuentan con una red de gasoductos que conectan los centros productores con las diferentes ciudades del país. A mediados de los años noventa esas empresas transportaban, respectivamente, alrededor del 65 y el $35 \%$ del gas natural producido en Argentina.

La privatización de las redes de distribución de Gas del Estado implicó su venta a ocho empresas distintas: Metrogas, Gas Natural BAN, Camuzzi Gas Pampeana, Camuzzi Gas del Sur, Litoral Gas, Centro, Cuyana y Gasnor. Las tres primeras representan el 54\% del gas distribuido en el país.

El transporte y la distribución de gas natural son considerados como actividades de servicio público, por lo cual el Estado ejerce una importante función reguladora, encargada al Ente Nacional Regulador del Gas (ENARGAS) en aspectos relacionados con las concesiones, acceso a las redes de transporte y distribución, tarifas y protección del consumidor.

\section{Bolivia: La prioridad de la exportación de gas natural}

Con reservas de unos $110000 \mathrm{MMm}^{3}$ Bolivia ocupa el séptimo lugar en América Latina. El gas natural tiene una gran importancia en la producción de energía primaria (58\% del total), seguido por el petróleo (24\%) y la hidroenergía y la leña (6 y 7\%).

La mayor parte de la producción de gas natural no se consume en el país, sino que se exporta a Argentina y a Brasil; además, hay compromisos de abastecimiento con Paraguay, aunque en bastante menor medida. Es por ello que la participación del gas natural en la oferta nacional de energía primaria alcanza a sólo $25 \%$ mientras que el petróleo representa el $42 \%$.

Como resultado del proceso de capitalización de YPFB —en 1996 y principios de 1997- las empresas privadas controlan actualmente toda la producción de gas natural en virtud de contratos de riesgo compartido con YPFB.

La nueva Ley de Hidrocarburos ( $\mathrm{N}^{\circ} 1689$, de abril de 1996) establece las modalidades de desarrollo de la industria de gas natural, las características del ente regulador y los mecanismos y modalidades de regulación del mercado; se norma tanto la exportación como el consumo interno, con una clara orientación a favor de las exportaciones. La Ley determina que los productores deberán satisfacer la demanda de gas natural 
que deriva de los compromisos de exportación del Estado boliviano.

La construcción del gasoducto Santa Cruz-São Paulo es uno de los proyectos más importantes de Bolivia de los últimos 20 años y prevé el aprovisionamiento de $105000 \mathrm{MMm}^{3}$ (3.7 billones de pies cúbicos). El gasoducto, de $3700 \mathrm{~km}$ de longitud, requiere una inversión de 2000 millones de dólares. El acuerdo tiene una duración de 20 años. En los primeros ocho años se exportarían $8 \mathrm{MMm}^{3}$ diarios y en los 12 años restantes $16 \mathrm{MMm}^{3}$ diarios, lo cual hace un total de $93000 \mathrm{MMm}^{3}$ para todo el período.

Se proyecta construir un gasoducto a Paraguay. En septiembre de 1996, ambos gobiernos firmaron un contrato para la exportación de gas natural por 2.4 $\mathrm{MMm}^{3}$ diarios. El volumen subiría hasta $3.3 \mathrm{MMm}^{3}$ en un plazo de 10 años.

Se trata de convertir a Bolivia en un centro de conexión de los proyectos de integración energética del gas natural. También se desarrollará el mercado interno, siempre y cuando ello no implique postergar la integración energética regional.

\section{Colombia: Hacia la masificación del consumo de gas natural}

Las reservas de gas natural de Colombia, con 214000 $\mathrm{MMm}^{3}$, la sitúan en cuarto lugar entre los países de la región. El gas natural ocupa el tercer lugar entre los productos de energía primaria de Colombia con cerca del $10 \%$ del total; la participación del petróleo es de casi $49 \%$ y el carbón mineral aporta el $26 \%$. Luego vienen la leña y la hidroenergía con el $7 \%$ y el $5 \%$.

La producción de gas natural $\left(7674 \mathrm{MMm}^{3}\right.$ en 1995) se destina íntegramente al consumo interno. El petróleo aporta $43 \%$ de la oferta total de energía primaria, y el gas natural $13 \%$, proporción similar a la del carbón (13.4\%) y la leña (13.3\%). Los productores de gas natural han suscrito contratos de asociación para entregar el producto a un precio determinado a ECOPETROL, empresa que determina las formas de transporte y distribución del producto en el mercado interno.

Desde 1991, Colombia busca incrementar el consumo interno de gas natural. Para ello, aprobó el programa para la masificación del consumo de gas cuyo objetivo principal es promover una matriz de consumo de energía más eficiente mediante la sustitución por gas de recursos energéticos de alto costo. Específicamente, se pretende masificar el consumo de gas propano aumentando la producción y la importación del combustible; optimizando el uso de las reservas disponibles de gas natural con la construcción de una red troncal de transporte de cobertura nacional; promoviendo la participación privada en las diferentes inversiones que prevé el plan, y logrando la equivalencia entre los precios y los costos reales de producción y prestación de los servicios.

La empresa estatal de petróleo, ECOPETROL, está encargada de desarrollar el programa de masificación del consumo de gas. En 1992, el Ministerio de Minas y Energía aprobó el plan general de transporte de gas natural, según el cual ECOPETROL debe desarrollar la red troncal nacional. Con esta red se abasteció a 517000 consumidores en 1995 y se espera llegar a 2 millones en el año 2000, con una inversión total estimada en unos 3 mil millones de dólares, a lo que debe agregarse la instalación de plantas termoeléctricas que serán alimentadas con gas natural según se prevé en el plan de expansión de energía eléctrica 1995-2007.

La estrategia gasífera de Colombia se basa en la siguiente división de tareas:

i) El sistema de transporte será desarrollado por ECOPETROL de manera directa o a través de inversiones privadas por medio de sistemas conocidos como el вот (Build-Operate-Transfer) o similares, y por concesiones otorgadas por el Ministerio de Minas y Energía.

ii) La construcción y operación de las redes de distribución urbana estarán a cargo de empresas privadas o mixtas, en las que pueden participar los departamentos o municipios.

iii) Se creará una nueva entidad encargada de la administración del sistema de transporte y comercialización del gas con participación del sector privado.

iv) Se instituirá un sistema de regulación especial así como una legislación independiente para la industria del gas natural.

Colombia proyecta un aumento considerable del mercado interno del gas natural, aprovechando las grandes reservas del país. ECOPETROL tiene un papel importante que cumplir, pues se encargará de construir los gasoductos y promover la iniciativa privada. Ya está en vigencia la legislación para regular el transporte y la distribución de gas natural.

\section{Chile: Dependencia energética y sustitución de fuentes}

Las reservas de gas natural de Chile ascienden apenas a $48000 \mathrm{MMm}^{3}$. El gas natural ocupa el segundo lugar en la producción de energía primaria con $24 \%$ del total; le aventaja la leña con poco más del $38 \%$. La 
hidroenergía aporta el 20\%, el carbón mineral el 11\% y el petróleo un poco menos del $7 \%$.

Para satisfacer las necesidades energéticas del mercado interno, Chile importa alrededor de 180000 barriles diarios de petróleo; esa fuente cubre el $46 \%$ de la oferta total de energía primaria, seguida de la leña y el carbón mineral con $19 \%$ y 15\%, respectivamente. El gas natural y la hidroenergía contribuyen con un $10 \%$ cada uno.

Chile se ha propuesto aumentar el consumo interno de gas natural, basándose en la importación de gas natural de Argentina. En julio de 1995, los gobiernos de Chile y Argentina suscribieron un protocolo por el cual se liberaliza el intercambio de gas natural, lo que implica que no se imponen restricciones para que productores y compradores de las dos naciones negocien libremente volúmenes, transporte, precios, lugar de origen y condiciones de los correspondientes contratos comerciales. ${ }^{5}$

El desarrollo de la industria de gas natural en Chile está enteramente en manos de la empresa privada, y en ese proceso la integración gasífera con Argentina cumple un papel fundamental. El gasoducto pionero entre Chile y Argentina fue el de Tierra del Fuego, tendido en virtud de un consorcio formado por YPF y Bridas de Argentina y Chauvco de Canadá. ${ }^{6}$

En los últimos años se han construido tres gasoductos entre Argentina y Chile. En agosto de 1997 se inauguró el gasoducto de propiedad de GasAndes, consorcio integrado por las empresas argentinas Techint y Compañía General de Combustibles, Novacorp de Canadá y las chilenas Gasco y Chilgener. Este gasoducto, que abastece el mercado de Santiago, el más importante del país, introducirá grandes modificaciones en el balance energético chileno pues impulsará la construcción de centrales térmicas para la producción de electricidad, lo que favorecerá la sustentabilidad del proceso de aprovechamiento energético.

En febrero de 1997 se suscribió el contrato entre la empresa chilena ENDESA y el consorcio norteameri-

\footnotetext{
${ }^{5}$ Los vendedores y compradores negociarán y contratarán el precio de compraventa del gas, los volúmenes involucrados, las garantías necesarias y otras condiciones comunes a este tipo de contratos, así como el transporte de gas, incluido los gasoductos correspondientes, desde los puntos de entrega a los centros de consumo.

${ }^{6}$ El gas abastece la planta de Methanex Chile Limited, subsidiaria de Methanex Corporation del grupo Nova, que se encuentra en Punta Arenas en el Chile austral. El ducto tiene $109 \mathrm{~km}$ pero se añadirán otros $106 \mathrm{~km}$ entre Poseidón y Cabo Negro para transportar 2.9 millones de metros cúbicos diarios desde 1999. El despacho comercial se inició en enero de 1997. Véase Latinominería (varios números).
}

cano CMS Energy para construir un gasoducto entre la provincia argentina de Salta (Campo de Durán) y la provincia de Atacama, en el norte de Chile. El objetivo principal del proyecto es abastecer centrales térmicas y empresas mineras del norte, donde se concentra el $30 \%$ de la producción mundial de cobre. Se calcula que la inversión total asciende a 900 millones de dólares.

El gasoducto del Pacífico (GasSur) unirá los campos de Neuquén con la localidad de Bío-Bío, en el sur de Chile. Tendrá una longitud de $530 \mathrm{~km}$ y la inversión será de 400 millones de dólares. El consorcio, liderado por Nova International (Canadá), junto con Gasco, ypF y El Paso Energy, invertirá otros 44 millones de dólares en servicios de gas natural (transporte y comercialización), así como en GasSur, un sistema de distribución comercial y residencial para la ciudad de Concepción (Petroleum Economist, varios números).

\section{México: Participación privada en el transporte y distribución}

El consumo de energía primaria de México se caracteriza por el claro predominio del petróleo con $69 \%$ del total (OLADE, 1996b). El gas natural representa el 16\% y el gas asociado y el gas no asociado cerca del $3 \%$ del consumo total, mientras que los condensados contribuyen con $2 \%$. Así, en conjunto, los hidrocarburos representan el $90 \%$ del consumo nacional de energía primaria. El 10\% restante está constituido por la hidroenergía (3\%), leña (3\%), bagazo de caña (1\%) y otros tipos de energía (1\%).

El renglón más importante en el consumo de gas natural es la extracción de licuables, los consumos propios del sector, el empleo de combustible en la generación de electricidad y los consumos finales no energéticos. Otro rubro importante es el de consumo industrial, en el que destaca la demanda de la industria petroquímica. El gas natural no ha logrado penetrar en el sector residencial y su empleo en el transporte es nulo.

La totalidad de las importaciones y exportaciones de gas natural efectuadas por México provienen de los Estados Unidos o se dirigen a este destino.

La producción de gas natural sigue siendo un monopolio estatal a cargo de PEMEX, no habiéndose producido ningún cambio en ese régimen en lo que va de los años noventa. Sin embargo, se han producido importantes modificaciones en el transporte y la comercialización del gas natural. En 1995 el Congreso aprobó la Ley Reglamentaria del Artículo 27 Constitucional, permitiendo que el transporte, almacenamiento y 
distribución de gas natural puedan ser llevados a cabo - previa aprobación de la Comisión Federal de Electricidad (CFE) - por los sectores social y privado, que podrán construir, operar y ser propietarios de ductos, instalaciones y equipos, en los términos de las disposiciones reglamentarias, técnicas y de regulación que se expidan. Esta medida fue complementada con la asignación de nuevas funciones y atribuciones a la Comisión Reguladora de Energía y con la expedición del Reglamento de Gas Natural, en noviembre de 1995.

Los cambios en la legislación son indicativos del impulso que se le quiere dar al consumo interno de gas natural, que todavía sólo abastece el $4 \%$ del consumo de energía residencial y no ha penetrado aún en el transporte. Habría, pues, fundadas expectativas de una ampliación futura de la demanda interna.

La reforma no abarca los aspectos relacionados con la explotación y producción de gas natural, que seguirán siendo de exclusiva responsabilidad de PEMEX. Sin embargo, las empresas privadas podrán construir y poseer nuevos gasoductos como complemento de la red troncal de $12000 \mathrm{~km}$ de PEMEX. Las empresas privadas tendrán libre acceso a la red de PEMEX, así como ésta lo tendrá a los ductos de las empresas privadas. PEMEX estima que éstos y otros proyectos podrían generar inversiones privadas por unos 4000 millones de dólares en los próximos años.

La nueva política pone término al monopolio estatal del transporte y la distribución del gas natural en México. Presumiblemente PEMEX no participará en la construcción de nuevos ductos, salvo en casos excepcionales relacionados con la producción de petróleo y gas.

\section{Perú: Predominio de la iniciativa privada y desarrollo del mercado interno}

Las reservas de gas natural de Perú ascienden a $201000 \mathrm{MMm}^{3}$ y les corresponde el quinto lugar en América Latina. La mayor parte de las reservas son las de Camisea, en el Cuzco, cuya fase de explotación está por iniciarse. Se estima que las reservas de gas natural y condensados de Camisea podrían llegar a unos $340000 \mathrm{MMm}^{3}$ (12 billones de pies cúbicos) y 650 millones de barriles, respectivamente.

El gas natural tiene hoy escasa importancia en la producción de energía primaria en el Perú con una participación de poco menos del $2 \%$. La fuente principal es el petróleo, que representa el $50 \%$ seguido por la leña con $31 \%$ y la hidroenergía con $12 \%$; corresponde a los productos derivados de la caña de azúcar y otras fuentes el porcentaje restante.
La producción de gas natural es muy pequeña: unos $258 \mathrm{MMm}^{3}$ a mediados de los años noventa. ${ }^{7} \mathrm{Su}$ participación en la oferta total de energía primaria representa sólo $1.4 \%$ frente a una contribución del petróleo que alcanza al 53\% y a una participación de $28 \%$ y $11 \%$, respectivamente, de la leña y la hidroenergía.

La explotación del gas de Camisea es una pieza fundamental de la política energética peruana ya que permitirá aumentar las reservas de gas natural y de condensados, impulsar la reconversión energética hacia combustibles más limpios y generar divisas por las probables exportaciones.

En mayo de 1996 se firmó un contrato de licencia, por cuarenta años, con el consorcio SHELL/MOBIL para la explotación del yacimiento de Camisea; en las tres etapas del contrato se proyectó una inversión total de 2400 millones de dólares. En este contrato el Estado se abstuvo de participar en la inversión y dejó la ejecución en manos del consorcio.

A mediados de julio de 1998, el consorcio SHELL/ MOBIL, al término de la primera etapa y tras arduas negociaciones entre las partes, decidió no continuar con la segunda etapa, con lo cual el contrato se dio por terminado. El consorcio consideró que, en las actuales condiciones, el proyecto de Camisea otorgaba una rentabilidad del $8.4 \%$ para la inversión en el proyecto, la cual no era aceptable, pues no le permitiría la recuperación de la inversión en los plazos deseados.

Para seguir adelante con la segunda etapa, el consorcio demandaba una serie de nuevos incentivos, como la aceptación gubernamental de un precio para el gas natural que no se ajustaba a lo establecido en el contrato; la participación en la distribución del gas en Lima; la posibilidad de exportar gas a Brasil mediante la interconexión con el gasoducto Santa Cruz-São Paulo; y reformas de la legislación eléctrica peruana para garantizar un precio del gas natural que le permitiera competir con otros combustibles en el abastecimiento de energía a las centrales termoeléctricas. En agosto de 1998 se formó una Comisión de Alto Nivel encargada de sacar adelante una licitación internacional para el desarrollo de este recurso, lo cual se prevé para septiembre de 1999.

En 1998 comenzó la producción de gas natural de los campos de Aguaytía. La inversión ejecutada permitirá la explotación de $6314 \mathrm{MMm}^{3}$ (223 000 millones de pies cúbicos). Se estima que la producción anual

\footnotetext{
${ }^{7}$ La mayor parte de la producción está destinada al consumo de la propia industria petrolera, abasteciendo también a la ciudad de Talara en el norte del país.
} 
será de $569 \mathrm{MMm}^{3}$ cúbicos $\left(1.58 \mathrm{MMm}^{3}\right.$ diarios) y que se obtendrán además 4000 barriles diarios de gas licuado de petróleo (GLP). El gas será usado para operar dos centrales termoeléctricas: una para la ciudad de Pucallpa y la otra en Aguaytía. Esta última, con capacidad de $140 \mathrm{Mw}$, se enlazará con el sistema eléctrico interconectado centro-norte (SICN).

Al momento de redactar este artículo no existía un marco regulatorio para la industria del gas natural en sus fases de producción, transporte, distribución y comercialización. En diciembre de 1996 se promulgó la Ley 26734 que creó el Organismo Supervisor de la Inversión en Energía (OSINERG); su función es fiscalizar el cumplimiento de las disposiciones jurídicas y técnicas relacionadas con las actividades de los subsectores de electricidad e hidrocarburos, así como de las normas de conservación y protección del medio ambiente. Sin embargo, entre sus atribuciones no está la de fijar las tarifas para el transporte y distribución del gas natural, ni la de otorgar concesiones.

\section{IV \\ El auge de las inversiones en gas natural y la integración energética regional}

En América Latina el gas natural se ha usado predominantemente en la industria, sobre todo en la petroquímica, salvo en Argentina donde, desde hace muchos años, se registra un uso comercial y residencial importante. El gas natural no era utilizado para la generación de energía eléctrica, pues predominaban las centrales hidroeléctricas, y las térmicas utilizaban combustibles derivados del petróleo (fuel oil) y carbón.

En los últimos años, ha ido creciendo significativamente la demanda de gas natural para la generación de energía eléctrica. Se estima que los países de la región requerirían más de $90 \mathrm{GW}$ de potencia en el período 1995-2005 y de 24 GW para el período 20052010, que deberán ser abastecidos en su mayoría por el sector privado (Kurtz, 1997). Esta demanda podría ser satisfecha con centrales térmicas a gas natural, considerando sobre todo que serían muy limitadas las posibilidades de desarrollo de proyectos hidroeléctricos. ${ }^{8}$ Se espera, asimismo, que la construcción de las centrales térmicas a gas natural contribuya a reducir las tarifas de electricidad, pues ese combustible es más

\footnotetext{
${ }^{8}$ Uno de los usos más importantes del gas natural estará estrechamente ligado a la generación de energía eléctrica en todo el mundo. El pronóstico de inversiones eléctricas mundiales asciende a 2.28 billones de dólares para el período 1995-2010, de los cuales cerca de dos terceras partes serán realizadas en los países en desarrollo. Para América Latina, el monto de la inversión prevista en proyectos eléctricos es de 203000 millones de dólares para el mismo período, de los cuales 23000 millones corresponderían a proyectos de centrales térmicas a gas natural (Estados Unidos, Departamento de Energía, 1998).
}

barato que el fuel oil que se usa en las centrales térmicas que suelen abastecer al mercado en las horas de punta.

La preferencia por el gas natural se debe, entre otros motivos, al alto costo de las obras de generación hidroeléctrica; a la nueva política de los organismos multilaterales de desarrollo (Banco Mundial y Banco Interamericano de Desarrollo) que supone el virtual cese del financiamiento de proyectos hidroeléctricos; a las innovaciones tecnológicas en las centrales térmicas de ciclo combinado, de construcción más rápida y de menor costo que las centrales hídricas; a las mejoras en los sistemas de transporte y distribución del gas natural y a la menor contaminación del medio ambiente que produce el gas natural, en relación con el carbón y el fuel oil.

Los planes nacionales para elevar el consumo de gas natural coinciden con diversas iniciativas que impulsan una mayor integración energética regional, como la proliferación de gasoductos que conectan países productores con países consumidores.

Los proyectos de inversión de gas natural identificados en la región ascienden a más de 29000 millones de dólares (cuadro 2). En su mayoría, se trata de inversiones en gasoductos, ya sea internacionales o nacionales. En esta cifra no están incluidas las inversiones en proyectos relacionados, como la construcción de centrales térmicas o el desarrollo de complejos petroquímicos (la excepción la constituyen los proyectos petroquímicos en Argentina y en Trinidad y Tabago). 
CUADRO 2

América Latina: Proyectos de gas natural

(Millones de dólares)

\begin{tabular}{|c|c|c|c|c|}
\hline Proyectos & Países & Inversión total & Longitud & Avance \\
\hline \multicolumn{5}{|c|}{ Proyectos de integración regional } \\
\hline GasAndes $^{\mathrm{a}}$ & Argentina-Chile & 350 & $450 \mathrm{~km}$ & Ejecutado \\
\hline Atacama $^{\mathrm{b}}$ & Argentina-Chile & 900 & 914 & En ejecución \\
\hline GasSurc & Argentina-Chile & 400 & 530 & En ejecución \\
\hline Tierra del Fuego-Cabo Negro & Argentina-Chile & 70 & & 1999 \\
\hline Gaucho $^{\mathrm{d}}$ & Argentina-Chile & 200 & 440 & En ejecución \\
\hline Buenos Aires-Montevideo & Argentina-Paraguay & 135 & 200 & En ejecución \\
\hline $\operatorname{Mega}^{\mathrm{e}}$ & Argentina & 500 & 600 & En ejecución \\
\hline Mercosur & Argentina-Bol.-Bra. & 3100 & 1500 & Factibilidad \\
\hline Santa Cruz-São Paulo & Bolivia-Brasil & 2000 & 1350 & En ejecución \\
\hline Bolivia-Paraguay & Bolivia-Paraguay & 300 & 1000 & Factibilidad \\
\hline Subtotal & & 7955 & & \\
\hline \multicolumn{5}{|l|}{ Proyectos nacionales } \\
\hline Petroquímica Bahía Blanca & Argentina & 700 & & En ejecución \\
\hline Polisur & Argentina & 250 & & En ejecución \\
\hline Plan de masificación & Colombia & 3000 & & En ejecución \\
\hline Distribución de gas natural & México & 4000 & & En ejecución \\
\hline Camisea & Perú & 2500 & & Factibilidad \\
\hline Aguaytía & Perú & 250 & 215 & Ejecutado \\
\hline Trinidad y Tabago & Trinidad y Tabago & 5000 & & \\
\hline Proyecto Cristóbal Colón & Venezuela & 5500 & & Factibilidad \\
\hline Subtotal & & 21200 & & \\
\hline Total & & 29155 & & \\
\hline
\end{tabular}

Fuente: Elaboración propia sobre la base de los resultados de la investigación.

a Mendoza-Santiago

b Salta-Atacama-Mejillones

c Neuquén-Bío Bío d Entre Ríos-Uruguayana-Porto Alegre

e Neuquén-Bahía Blanca. Comprende exportación a Brasil

\section{$\mathrm{V}$}

\section{Los regímenes regulatorios de la industria de gas natural en América Latina}

\section{Principios y objetivos de la regulación}

Al momento de redactar este informe, sólo Argentina, Bolivia, Colombia, Chile y México contaban con leyes para regular los mercados de transporte y distribución de gas natural. En Perú y Venezuela aún no han sido promulgados los dispositivos correspondientes. ${ }^{9}$

\footnotetext{
${ }^{9}$ Para el desarrollo de esta sección se han consultado los dispositivos legales y las publicaciones de los entes y ministerios de los países analizados. La lista completa puede encontrarse en Campodónico (1998).
}

Los principios de la regulación gasífera son similares en casi todos los países analizados. Se trata de dar adecuada protección a los usuarios finales, teniendo en cuenta que algunas fases de la industria del gas (transporte y distribución) constituyen monopolios naturales. En estos casos, el Estado debe intervenir para garantizar la libre competencia, impedir los eventuales abusos de una posición dominante en el mercado, y favorecer la continuidad y calidad de la prestación de los servicios. Cuando no es posible que estos se ofrezcan en condiciones de competencia es indispensable que intervenga el Estado para que las operaciones sean eficientes, lo que implica la optimización de la relación calidad-precio. 
Los programas de acción de los entes reguladores se basan en los principios de la regulación gasífera que comprenden, entre otros: una utilidad justa o razonable; competencia; eficiencia y racionalidad; optimización de la calidad; confiabilidad, seguridad y continuidad del servicio; acceso sin discriminaciones; y cobertura máxima. Alrededor de estos principios los marcos reguladores contemplan objetivos como:

- Favorecer los intereses y derechos de los usuarios mediante el mejoramiento de la calidad del servicio público y su disposición final para asegurar una mejor calidad de vida a los usuarios;

- Promover la competitividad de los mercados de oferta y demanda de gas natural evitando el abuso por una posición dominante en el mercado;

- Propender a una mejor operación, confiabilidad, igualdad, libre acceso, no discriminación y uso generalizado de los servicios e instalaciones de transporte y distribución de gas natural;

- Regular el transporte y distribución de gas natural, asegurando que las tarifas que se apliquen sean justas y razonables;

- Asegurar la prestación continua e ininterrumpida de los servicios, sin excepción alguna, salvo cuando existan razones de fuerza mayor o caso fortuito o de orden técnico o económico que lo impidan;

- Elevar los índices de seguridad y reducción de incidentes vinculados con las prestaciones;

- Estimular el uso racional del gas natural velando por la adecuada protección del medio ambiente;

- Promover la inversión para asegurar el suministro a largo plazo.

Las legislaciones vigentes no incluyen un tratamiento explícito de los asuntos relacionados con la equidad social y la participación de los usuarios, salvo en Colombia, donde la legislación señala que debe ampliarse permanentemente la cobertura aplicando mecanismos que compensen la insuficiente capacidad de pago de los usuarios y debe garantizarse a los usuarios el acceso adecuado a los servicios y la participación en su gestión y fiscalización.

\section{Carácter de servicio público}

Todos los países analizados establecen una distinción entre las fases de producción y las de transporte y distribución. La producción de gas natural se considera de interés general, y se rige de acuerdo con las normas convencionales del mercado y la competencia, por lo que no está sujeta a regulación. En términos concretos, esto significa que el precio del gas natural en boca de pozo - llamado también precio del gas de primera mano- se rige por leyes de la oferta y la demanda.

El transporte y la distribución del gas natural son considerados actividades de servicio público y por lo tanto, son objeto de regulación por parte del Estado.

\section{Establecimiento de entes reguladores}

En todos los países que cuentan con marcos regulatorios se han creado entes reguladores cuya constitución es variable según sea la forma de organización del Poder Ejecutivo. Sin embargo, tienden en general a contar con autonomía administrativa. En algunos casos están adscritos a ministerios o secretarías de Estado (Argentina, Colombia), en otros forman parte de los sistemas nacionales de regulación (Bolivia) y en Chile, la regulación es ejercida por una comisión interministerial. Presentan también particularidades de financiamiento. En algunos casos dependen del presupuesto general, pero en otros (Bolivia y Colombia) se financian con aportes de las empresas reguladas.

En Argentina se constituyó el Ente Nacional Regulador del Gas (ENARGAS) ubicado en el ámbito del Ministerio de Economía, Obras y Servicios Públicos. En Bolivia existe la Superintendencia de Hidrocarburos, perteneciente al Sistema de Regulación Sectorial (SIRESE) que es parte del Poder Ejecutivo, bajo la tuición del Ministerio de Hacienda y Desarrollo Económico. En Colombia existe la figura jurídica de Empresa de Servicio Público que tiene una legislación específica. En el sector actúa la Comisión de Regulación de Energía y Gas Combustible, adscrita al Ministerio de Minas y Energía como una unidad administrativa especial, con independencia administrativa, técnica y patrimonial. Además, se constituyó la Superintendencia de Servicios Públicos Domiciliarios que, por encargo de la Presidencia de la República, ejerce el control, inspección y vigilancia de las entidades que prestan esos servicios. En Chile no existe un ente regulador especializado en la industria del gas natural. La Comisión Nacional de Energía, dirigida por un Ministro Presidente de la Comisión, se ocupa de la regulación en tanto que la Superintendencia de Electricidad y Combustibles, adscrita al Ministerio de Economía, ejerce funciones de fiscalización. En México, la Comisión Reguladora de Energía (CRE) tiene a su cargo la regulación del gas; es un organismo descentralizado de la Secretaría de Energía, que a su vez es una dependencia del Poder Ejecutivo.

Las funciones de los entes reguladores son similares en casi todos los países analizados; a saber: 
- Otorgar concesiones y licencias a los transportistas y distribuidores de gas natural y declarar o disponer su caducidad o revocatoria.

- Vigilar el cumplimiento de las obligaciones y derechos de los concesionarios y licenciatarios.

- Proteger los derechos de los consumidores, cargadores, concesionarios y productores.

- Asegurar el cumplimiento de las disposiciones antimonopolistas y de defensa de la competencia.

- Garantizar el acceso libre e indiscriminado a los ductos.

- Regular, fijar tarifas y promover la eficiencia y la continuidad de los servicios de transporte y distribución.

- Realizar todos los actos inherentes y necesarios para el cumplimiento de sus funciones de acuerdo a las normas vigentes.

Sin embargo, los entes reguladores presentan también características y atribuciones particulares. En Chile, las tarifas de transporte y distribución se fijan libremente y no están sujetas a regulación, salvo en el caso de usuarios de menos de 100 Gigajoule. En México, el organismo que fija las tarifas de transporte y distribución es el Comité de Productos Petrolíferos, Gas Natural y Productos Petroquímicos, integrado por una serie de organismos entre los que se encuentra la Secretaría de Energía.

\section{Prohibición de la integración vertical}

El tema de la integración vertical ha preocupado a los encargados de la regulación. Según el tipo de actividad y la dimensión de los mercados objeto de regulación, se considera que la integración vertical podría generar situaciones que afectarían a la competencia.

En la regulación regional de la industria del gas natural sólo en Chile no existe prohibición expresa. La integración vertical entre las actividades de producción, transporte y distribución se prohíbe explícita y categóricamente sólo en Argentina y Colombia, mientras que en Bolivia y México se prohíbe con excepciones.

La legislación de Bolivia autoriza la integración vertical para proyectos y operaciones en zonas aisladas que no sean financiables ni económicamente rentables sin ella; o cuando sea necesario para el desarrollo de nuevos mercados internos de distribución de gas natural por redes, cuya eficiencia depende de la integración vertical.

En México, la Comisión Reguladora de Energía (CRE) puede autorizar la integración vertical cuando, a su juicio, ésta redunde en beneficios de eficiencia y rentabilidad en la prestación del servicio o cuando sea estrictamente necesaria por no existir la infraestructura de transporte requerida para desarrollar el mercado de una zona geográfica determinada y no haya otros interesados en acometer el proyecto de transporte o distribución.

\section{Libre acceso a las redes de transporte y distribución}

En Argentina, Bolivia, Colombia, Chile y México se establece que los concesionarios, licenciatarios o permisionarios deberán permitir a los usuarios el acceso abierto, sin ningún tipo de discriminaciones, a los servicios de sus respectivos sistemas. Sin embargo, la legislación vigente incluye algunas limitaciones respecto del libre acceso que tienen relación con la capacidad disponible o no contratada y con la utilización efectiva de la capacidad contratada. Se precisa que, en cualquier caso, el usuario potencial debe celebrar el contrato correspondiente para la prestación del servicio.

En Colombia, están en vigencia códigos de transporte y de distribución que establecen los mecanismos para que los productores, comercializadores, grandes consumidores y distribuidores puedan ejercer el principio del libre acceso a las redes.

\section{Formación de los precios en boca de pozo}

En la mayoría de los países analizados el precio del gas natural en boca de pozo es de interés general y por lo tanto, no está sujeto a regulación. Sin embargo, existen problemas para determinar el precio del gas ya que, a diferencia del petróleo, no existe para el gas natural una cotización internacional de referencia. En algunos casos, se toma como referencia el precio internacional del fuel oil, combustible que puede ser sustituido por el gas natural. Podría suceder que, a medida que aumentan las reservas de gas y se incrementan las posibilidades de sustitución de otros combustibles, el costo de oportunidad se encuentre más ligado a los costos medios de producción, transporte y distribución del gas.

En Argentina, el precio del gas natural en boca de pozo es de interés general, pues resulta del libre juego de la oferta y la demanda, mientras que en Bolivia, donde la mayor parte del gas es exportado a Argentina, el precio es concertado en negociaciones entre ambos países. Lo propio ocurrirá con las futuras 
exportaciones a Brasil a través del gasoducto Santa Cruz-São Paulo. ${ }^{10}$

En Colombia, la ley establece que habrá libertad de precios cuando exista una competencia efectiva entre los proveedores y no haya una situación de dominio del mercado, la que será calificada por el ente regulador. En la transición, si bien pueden aplicarse varias modalidades para la fijación del precio, ECOPETROL compra el gas natural a sus asociados al precio internacional del fuel oil. ${ }^{11}$

En Chile, los precios son decididos libremente por los exportadores e importadores, de acuerdo con lo determinado en el protocolo de complementación económica entre Argentina y Chile, suscrito en 1995.

En México, los precios del gas seco son equiparados con los del sur de Texas, por la posibilidad de acceso a ese mercado, más los costos de transporte y de servicio y el impuesto al valor agregado. Para la fijación de los precios internos, se aplica el índice de precios internos de los combustibles y se vinculan con una canasta de precios del mercado de Estados Unidos, más el costo de transporte en línea con el sistema de paridad de importaciones.

En Perú, las autoridades todavía no han legislado sobre la materia. Sin embargo, en los contratos de petróleo y gas natural se ha determinado la forma de valoración en cada caso, pero en general rige el precio de mercado. Así, por ejemplo, en el contrato con el consorcio Shell/Mobil para la explotación del gas de Camisea — suscrito en mayo de 1996 - se estipula que el valor del gas natural se calculará según el precio realizado que deberá reflejar el precio de mercado del gas producido en el área del contrato. El precio realizado se define como el precio efectivamente pagado

${ }^{10}$ En 1995, el precio medio anual en boca de pozo en Argentina fue de 1125 dólares por millón de British Thermal Units (MMBTU), lo que equivale a 1.20 dólares por mil pies cúbicos $(\mathrm{mpc})$. El precio del gas que se exportará de Bolivia a Brasil será de 0.90 dólares/ MMBTU. Para los nuevos contratos o cuando los contratos existentes concluyan - dentro de cinco años - el precio de venta a las plantas termoeléctricas, industriales y empresas distribuidoras de gas natural por redes, se calculará de acuerdo con el siguiente procedimiento: Primero se determinará el precio ponderado de exportación a la Argentina y Brasil, puesto en boca de pozo. A este monto se le añadirá la tarifa de transporte para el consumo interno. Este nuevo valor se multiplicará por el factor 1.1494 y el resultado será el precio de venta a productores y cargadores en el punto de entrega del mercado interno.

${ }^{11}$ Mientras no existan precios libres, el precio máximo inicial en el nodo de entrada al sistema nacional de transporte será de 1.30 dólares/MMBTU (1.391 dólares/mpc). Este precio se modificará semestralmente a partir del primero de enero de 1996, a base de una fórmula que considera la variación del índice de precios para el crudo estándar cotizado en el New York Mercantile Exchange (NYMEX). por el comprador al contratista de gas natural, en este caso el consorcio Shell/Mobil. ${ }^{12}$

En Venezuela, los precios reflejan el costo de oportunidad y varían según la ubicación geográfica. ${ }^{13}$ A modo de referencia, debe indicarse que en Estados Unidos los precios son libres y registran fluctuaciones significativas según la estación del año. ${ }^{14}$

\section{Regulación de tarifas de transporte y distribu- ción}

En Argentina, Bolivia, Colombia y México los entes reguladores fijan las tarifas de transporte y distribución de gas natural; en Chile, en cambio, sólo regulan las tarifas para usuarios de menos de 100 Gigajoules. En los países que regulan, la tarifa que abona el usuario final es el resultado del precio del gas natural, negociado en el mercado mayorista, más las tarifas por los servicios de transporte y distribución. Existen, sin embargo, diferentes métodos de cálculo de las tarifas de transporte y distribución.

En Argentina, ENARGAS aprueba las tarifas y adopta una tarifa máxima o precio tope, ajustable por un índice de precios y un factor de productividad. Al ser tarifas máximas, el prestador del servicio puede disminuirlas según su conveniencia comercial (mantener o ampliar mercados) sin autorización de ENARGAS, teniendo siempre en vista la recuperación de los costos.

ENARGAS debe garantizar a los transportistas y distribuidores, que operen de manera adecuada, la obtención de ingresos suficientes para satisfacer todos los costos operativos razonables del servicio, impuestos, amortizaciones y una rentabilidad razonable. El concepto de rentabilidad razonable se define, en el marco jurídico vigente en la Argentina, como la que podría obtenerse en otras actividades de riesgo equiparable; debe, además, guardar relación con cierto grado de eficiencia y prestación satisfactoria de los servicios.

Las tarifas fijadas por ENARGAS deben tomar en cuenta las diferencias que pudieran existir entre los distintos tipos de servicios en cuanto a su forma de

\footnotetext{
${ }^{12}$ En el Plan Referencial de Electricidad del Ministerio de Energía y Minas se hace un pronóstico sobre los precios del gas natural de Camisea, así como de Aguaytía y Talara. Estos son 1.5 dólares por mpc en boca de pozo en Camisea; 2.5 dólares/mpc puesto en Lima; 2.5 dólares/mpc para Aguaytía, y 2.0 dólares/mpc en Talara.

${ }^{13}$ Se estima que en José, el precio sería 0.53 dólares/mpc y de 0.642 dólares/mpc en Puerto Ordaz (OLADE, 1997b).

${ }^{14}$ Los precios en boca de pozo en Estados Unidos fueron de 1.59 dólares/mpc en promedio en agosto de 1996 (Oil \& Gas Journal, varios números).
} 
prestación, ubicación geográfica, distancia relativa de los yacimientos y cualquier otra modalidad que ese ente califique como atinente. Si bien el precio de venta del distribuidor al consumidor incluye los costos de adquisición y otros involucrados en la prestación del servicio, corresponde al ente regulador asegurar a los consumidores el costo mínimo compatible con la seguridad del abastecimiento.

En México, el sistema tarifario adoptado es también el de tarifa máxima, siendo propuesta a la Comisión Reguladora de Energía por los interesados en prestar el servicio. Las tarifas iniciales que se adopten deben permitir que éstos utilicen racionalmente los recursos y en la eventualidad de su ajuste, garantizar ingresos suficientes para cubrir los costos adecuados de operación y mantenimiento aplicables al servicio, los impuestos, la depreciación y una rentabilidad razonable. El mecanismo no asegura pues, necesariamente, los ingresos, costos o rentabilidad esperados por quienes soliciten permiso para prestar el servicio.

El método de fijación de tarifas no es aplicable cuando, a juicio de la Comisión Reguladora de Energía, hay condiciones de competencia efectiva. Por lo tanto, los concesionarios pueden solicitar al ente regulador que se pronuncie sobre la vigencia efectiva de la competencia.

En Bolivia se aplica la regulación mediante tasa de retorno. Las tarifas para el transporte de hidrocarburos y sus derivados por ductos, así como para la distribución de gas natural por redes, son aprobadas por la Superintendencia de Hidrocarburos del Sistema de Regulación Sectorial.

El mecanismo establecido permite que la tarifa garantice una determinada rentabilidad de la inversión realizada por el concesionario. Para estos efectos, el ente regulador debe asegurar el costo más bajo a los usuarios del sistema de transporte de hidrocarburos y sus derivados, así como de la distribución de gas natural por redes, precautelando la seguridad y continuidad del servicio. El costo debe asegurar a los concesionarios - con una administración racional y prudente- ingresos suficientes para cubrir todos sus gastos de operación, impuestos (con excepción del impuesto a la remisión de utilidades al exterior), depreciación y costos financieros para obtener una rentabilidad razonable sobre su patrimonio neto. Corresponde además, al ente regulador estimular a los concesionarios para que incrementen la eficiencia de sus operaciones.

En Colombia no existe un sistema tarifario único. Si no hay una posición dominante en el mercado y por tanto, existe un régimen de competencia — situa- ción que debe ser calificada por el ente regulador- las empresas de servicio público de gas deciden libremente sus tarifas. En caso contrario se someten al régimen de regulación que prevé las modalidades de libertad vigilada y libertad regulada. La primera rige cuando existe competencia y la segunda cuando las condiciones del mercado exigen la intervención del ente regulador.

Las empresas se rigen por las fórmulas que define periódicamente el ente regulador para la fijación de tarifas. Sobre la base del estudio de costo, el ente puede establecer topes tarifarios máximos y mínimos, de cumplimiento obligatorio, y definir cuándo conviene aplicar los regímenes de libertad regulada o vigilada.

En Colombia se combinan los métodos de tarifa máxima - con la variante de tarifa mínima - y de tasa de retorno de la inversión.

La ley establece que la metodología para la fijación de las tarifas estará orientada por los criterios de eficiencia económica, neutralidad, solidaridad, redistribución, suficiencia financiera, simplicidad y transparencia; establece además que los criterios de eficiencia económica y suficiencia financiera tienen prioridad en la definición del régimen tarifario. Si se presentara contradicción entre ambos criterios, las tarifas se definen aplicando el criterio de suficiencia financiera, el que, a juicio del regulador, garantiza la eficiencia económica.

El régimen procurará que las tarifas se aproximen a lo que serían los precios de un mercado competitivo. Para estos efectos, las fórmulas de fijación de tarifas deben tener en cuenta no sólo los costos sino los aumentos de productividad esperados, que deben distribuirse entre la empresa y los usuarios, tal como ocurriría en un mercado competitivo. Así las fórmulas tarifarias no pueden trasladar a los usuarios los costos de una gestión ineficiente, ni permitir que las empresas se apropien de las utilidades provenientes de prácticas restrictivas de la competencia.

En los servicios públicos las fórmulas tarifarias deben reflejar siempre el nivel y la estructura de los costos económicos de prestar el servicio, así como la demanda.

Todos los consumidores tienen el derecho al mismo tratamiento tarifario si las características de los costos que ocasionan a las empresas prestatarias son iguales. El ejercicio de este derecho no debe impedir que las empresas de servicios públicos ofrezcan opciones tarifarias y que el consumidor escoja la que más le convenga. 
Al poner en práctica el régimen tarifario se adoptarán medidas para asignar recursos a fondos de solidaridad y redistribución, para que los usuarios de los estratos altos y los usuarios comerciales e industriales, ayuden a los usuarios de estratos bajos a pagar las tarifas de los servicios que cubran sus necesidades básicas.

Las fórmulas tarifarias deben garantizar la recuperación de los costos y gastos propios de operación, incluidos la expansión, la reposición y el mantenimiento, y permitir remunerar (método de la tasa de rentabilidad) el patrimonio de los accionistas en la misma forma que lo habría remunerado una empresa eficiente en un sector de riesgo comparable; utilizará para ello las tecnologías y sistemas administrativos que garanticen la mejor calidad, continuidad y seguridad del servicio.

Las fórmulas para la fijación de tarifas se elaborarán en forma tal que se facilite su comprensión, aplicación y control.

El régimen tarifario será explícito y completamente público para todas las partes involucradas en el servicio y para los usuarios.

\section{Subsidios cruzados y subsidios fiscales}

Las políticas aplicadas en los países analizados se diferencian en cuanto a la aplicación de subsidios. Se aprecian dos tendencias en los países de la región:

La prohibición de subsidios cruzados y la posibilidad de aplicar subsidios fiscales se advierten en Argentina, Bolivia, Chile y México. En Argentina están prohibidos los subsidios cruzados, pero pueden aplicarse subsidios fiscales o gubernamentales, siempre que estén previstos explícitamente en el presupuesto nacional. Estos pueden otorgarse a los usuarios residenciales del sur del país y a la denominada clase pasiva.

En Bolivia no se permiten subsidios cruzados, pero la jurisprudencia no especifica si pueden o no apli-

\section{VI}

\section{Conclusiones}

El aumento de la producción y el consumo de gas natural en todo el mundo, especialmente en los países en desarrollo (China, el Sudeste asiático y América Latina), es una característica de los mercados energéticos. Este crecimiento tiende a consolidarse a mediano y carse subsidios fiscales. En Chile tampoco están permitidos los subsidios cruzados. Sin embargo, en el caso de consumidores de menos de 100 Gigajoule, las tarifas pueden ser reguladas si se demuestra que el sistema tarifario permite obtener ingresos de explotación que otorguen una rentabilidad económica superior en $5 \%$ a la tasa de costo anual del capital.

Los subsidios cruzados no están permitidos en México. El otorgamiento de subsidios fiscales a través de las tarifas sólo podrá derivarse de disposiciones adoptadas por las autoridades competentes y deberán cubrirse con recursos que éstas registren para tales propósitos. Su otorgamiento no deberá afectar los ingresos de los permisionarios ni representar un costo adicional para los mismos. Su aplicación deberá ser transparente y quedar explícita en las tarifas que se cobren a los usuarios.

En Colombia están permitidos los subsidios cruzados con el objeto de beneficiar a los sectores de bajos ingresos. Las empresas de servicio público que suministren o comercialicen gas combustible los recaudarán, en nombre de los consumidores que abastecen, aportando al Fondo de Solidaridad y Redistribución de Ingresos de la Nación la suma que resulte de aplicar el factor pertinente del $20 \%$ al costo económico del suministro, en puerta de ciudad, según lo que disponga la Comisión de Regulación de Energía y Gas domiciliario. Los recursos de dichos fondo serán destinados a dar subsidios a los usuarios de estratos de bajos ingresos, como inversión social, en los términos que fije la ley. Los Fondos de Solidaridad y Redistribución de Ingresos creados por los concejos municipales reciben las transferencias de las empresas de servicios públicos y conceden los subsidios según las reglas establecidas por la ley.

La legislación de los países analizados no incluye referencias al régimen tributario, pudiendo inferirse que las empresas que operan en la industria del gas natural se someten al régimen tributario común.

largo plazo por diversos motivos: avance tecnológico, dispersión geográfica de las reservas, menor contaminación del medio ambiente que otros combustibles fósiles, crecimiento de los mercados energéticos en los países emergentes y consideraciones de seguridad geo- 
gráfica del abastecimiento, sobre todo en los países industrializados.

Las modificaciones de los regímenes jurídicos de hidrocarburos en el decenio de 1990 en los países de la región han tenido por objeto abrir y desregular los mercados a fin de atraer nuevos capitales de inversión. La abundancia de reservas de gas natural en varios de estos países permite proyectar la inversión en los próximos años en la importante cifra de 29000 millones de dólares.

El alto costo de exportación por vía marítima hace que el uso del gas natural esté orientado a satisfacer las necesidades energéticas de los mercados internos de los países de la región (centrales térmicas, consumo industrial y doméstico, desarrollo de la petroquímica) y a la integración energética regional gracias a la construcción de gasoductos interfronterizos.

En casi todos los países analizados, la producción de gas natural proviene de diversas empresas petroleras públicas y privadas, su precio no está regulado y se rige de acuerdo con la oferta y la demanda. Sin embargo, en muchos países el Estado interviene en la fijación de los precios en boca de pozo, debido a que se trata de una industria naciente y a que no existe un solo precio internacional que sirva como patrón para todos los mercados.

En el suministro de gas natural y de electricidad a los clientes finales el precio no se fija por la oferta y la demanda, sino que está regulado por el Estado. Esto se debe a que el transporte y la distribución del gas natural se basa en redes que constituyen un monopolio natural y a que ésta es una fuente energética que se considera servicio público. Estos dos aspectos imponen la regulación estatal para asegurar la libre competencia de suministro (recuérdese que antes de la reforma, en casi todos los países de la región los servicios públicos estaban reservados a la actividad empresarial del Estado), impedir los posibles abusos por gozar de una posición dominante en el mercado, y favorecer la continuidad y la calidad del servicio. Se considera que cuando no existen condiciones de competencia, el Estado debe intervenir para que las operaciones sean eficientes, lo que implica optimizar la relación calidadprecio.
La nueva legislación en materia de regulación de los países de la región otorga derechos de monopolio y otros derechos especiales a las compañías de electricidad y de gas natural en el contexto de las obligaciones del servicio público. El grado y forma de regulación varían considerablemente según quién sea el dueño de la empresa y cuáles sean las fuentes de generación de energía y las tradiciones administrativas del país, y también por consideraciones políticas.

En el decenio de 1990 se generalizó la existencia de entes reguladores en casi todos los países de la región; en algunos casos, éstos apenas tienen dos o tres años de funcionamiento, lo que quiere decir que están en la etapa de aprendizaje. Por ello, es de suma importancia que los gobiernos impulsen políticas de capacitación y que otorguen el apoyo necesario al desarrollo de las labores de estos entes.

Algunas de las áreas claves para la acción del gobierno y de los entes reguladores podrían ser las siguientes:

- Control de la explotación de los recursos naturales, en particular del petróleo y del gas natural.

- Regulación de las industrias de redes con un énfasis creciente en la promoción de la competencia; garantía del libre acceso a redes; manejo flexible de la integración vertical del transporte y la distribución, haciendo hincapié en un estricto control regulatorio; transparencia y equidad en la fijación de tarifas para el transporte y distribución de gas natural; protección de los derechos de los consumidores.

- Difusión de información y mayor educación de los usuarios energéticos.

- Intervención en los contratos de gran escala para la importación de gas natural.

- Refuerzo de las regulaciones ambientales relativas a la producción y uso de energía, lo que incluye el estudio de la viabilidad del uso de políticas impositivas como instrumentos activos de las políticas energéticas y ambientales.

- Investigación y desarrollo tecnológico.

- Promoción y mayor uso de energías renovables. 


\section{Bibliografía}

Campodónico, H. (1996): El ajuste petrolero: políticas empresariales en América Latina de cara al 2000, Lima, Ediciones DESCO (1998): La industria del gas natural y las modalidades de regulación en América Latina, Serie medio ambiente y desarrollo, $\mathrm{N}^{\circ}$ 9, Santiago de Chile, Comisión Económica para América Latina y el Caribe (CEPAL), División de Medio Ambiente y Desarrollo.

Estados Unidos, Departamento de Energía (1995): International Energy Outlook, 1995, Washington D.C.

(1998): International Energy Outlook, 1998, Washington D.C.

Kurtz, D. (1997): Natural Gas in Latin America: Development and Privatization, Londres Financial Times Energy Publishing.

Latin Finance (varios números): Miami, Florida.

Latinominería (varios números): Santiago de Chile, G\&T International, septiembre.

OCDE (Organización de Cooperación y Desarrollo Económicos), Organismo Internacional de Energía (1995): World Energy Outlook 1995, París.
Oil and Gas Journal (varios números): Oklahoma, Penn Well Publishing Company.

OLADE (Organización Latinoamericana de Energía) (1996a): Alternativas de integración en el mercado latinoamericano y caribeño del gas natural: estudios de casos, Quito, noviembre. (1996b): Alternativas de integración en el mercado latinoamericano y caribeño del gas natural: el caso de México, Quito, noviembre.

(1997a): Sistemas de información económica energética, Quito.

(1997b): Modernización, desincorporación de activos y libre comercio en la actividad de gas natural en América Latina y el Caribe, Quito, abril.

Perú, Ministerio de Energía y Minas (1997): Plan referencial de electricidad, Dirección General de Electricidad, Lima.

Petroleum Economist (varios números): Londres, Sedwick Energy Limited. 